\title{
Gagliardo-Nirenberg Inequality as a Consequence of Pointwise Estimates for the Functions in Terms of Riesz Potential of Gradient
}

\author{
Sudheer Khan, Wang Shu, Monica Abhidha \\ College of Applied Sciences, Beijing University of Technology, Beijing, China \\ Email address: \\ soudkayani@hotmail.com(S. Khan), shu.wang@bjut.edu.cn (Wang Shu), optimisticthinker@yahoo.com (M. Abhidha) \\ To cite this article: \\ Sudheer Khan, Wang Shu, Monica Abhidha. Gagliardo-Nirenberg Inequality as a Consequence of Pointwise Estimates for the Functions in \\ Terms of Riesz Potential of Gradient. Science Journal of Applied Mathematics and Statistics. Vol. 8, No. 5, 2020, pp. 53-58. \\ doi: $10.11648 /$ j.sjams.20200805.11
}

Received: July 1, 2020; Accepted: July 16, 2020; Published: September 21, 2020

\begin{abstract}
Our aim in this study is to give the Gagliardo-Nirenberg Inequality as a consequence of pointwise estimates for the function in terms of the Riesz potential of the gradient. Our aim here is to discuss boundedness of Reisz potential in term of maximal functions and to give the proof for Gagliardo-Nirenberg Inequality in term of Reisz potential. We will extend our result to discuss weak type estimate for Gagliaro-Nirenberg Sobolev inequality. Further, in this paper we are interested to extract Sobolev type inequality in terms of Riesz potentials for $\alpha$ is equal to one and to extend our work for weak type estimates when $p$ is equal to one.
\end{abstract}

Keywords: Gagliardo-Nirenberg Inequality, Hardy Littlewood Maximal Function, Riesz Potential, Sobolev Type Inequality

\section{Introduction}

The Hardy-Littlewood maximal function was considered as a classical tool in various areas such as potential analysis and harmonic analysis from years and later in Sobolev space theory and partial differential equations see [1, 4, 12], and [13]. Sobolev space plays a significant role in dealing with existence and regularity of solutions of Partial Differential Equations see $[5,8]$. The Hardy-Littlewood maximal function bridging between functional analysis, sobolev spaces and partial differential equations [7, 9, 11, 17]. Boundedness of Maximal function has been discussed earlier with different arguments such as, Boundedness and regularity of maximal functions on hardy-sobolev spaces discussed in [15]. Luiro generalized the original boundedness result and established the continuity of the centered maximal operator in $W^{1, p}\left(R^{d}\right), 1<p<\infty$ [13]. P. Hajlasz and J. Onninen proved the boundedness of the spherical maximal function in the Sobolev space $W^{1, p}(\Omega), \mathrm{p}>\frac{d}{d-1}$. For other arguments and related results, one can see $[10,16]$ and [18]. With the strong arguments over the boundedness of Hardy-Lilltewood Maximal function, our aim here is to discuss boundedness of Reisz potential in term of maximal functions and to give the proof for Gagliardo-Nirenberg Inequality in term of Reisz potential. We will extend our result to discuss weak type estimate for Gagliaro-Nirenberg sobolev inequality.

We start by recalling the definition of maximal function.

Let $B(y, a)=\left\{x \in R^{d}:|x-y|<a\right\}$ is an open ball having center at $y \in R^{d}$ and radius $a>0$ then

$M f: R^{d} \rightarrow[0, \infty]$ where $f \in L_{l o c}^{1}\left(R^{d}\right)$ is

$$
M f(y)=\sup _{a>0} \frac{1}{|B(y, a)|} \int_{B(y, a)}|f(x)| d x
$$

As from Lebesgue differentiation theorem, for all $y \in R^{d}$

$$
\begin{aligned}
|f(y)| & =\lim _{a>0} \frac{1}{|B(y, a)|} \int_{B(y, a)}|f(x)| d x \\
& \leq \sup _{a>0} \frac{1}{|B(y, a)|} \int_{B(y, a)}|f(x)| d x=M f(y)
\end{aligned}
$$

As maximal function approach involves in our theme of work, we will begin with some basic and obvious results.

Lemma 1: If $f \in L^{\infty}\left(R^{d}\right)$, then $M f \in L^{\infty}\left(R^{d}\right)$ and $\|M f\|_{L^{\infty}\left(R^{d}\right)} \leq\|f\|_{L^{\infty}\left(R^{d}\right)}$.

Proof: For any $a>0$ and for all $y \in R^{d}$ we can write 


$$
\begin{gathered}
\frac{1}{|B(y, a)|} \int_{B(y, a)}|f(x)| d x \leq \frac{1}{|B(y, a)|}\|f\|_{L^{\infty}\left(R^{d}\right)}|B(y, a)| \\
=\|f\|_{L^{\infty}\left(R^{d}\right)} \\
\sup _{a>0} \frac{1}{|B(y, a)|} \int_{B(y, a)}|f(x)| d x \leq\|f\|_{L^{\infty}\left(R^{d}\right)}
\end{gathered}
$$

Using (1.1), we have $M f(y) \leq\|f\|_{L^{\infty}\left(R^{d}\right)}$

Thus $\|M f\|_{L^{\infty}\left(R^{d}\right)} \leq\|f\|_{L^{\infty}\left(R^{d}\right)}$

It shows the maximal function will essentially bounded with the boundedness of original function and thus finite everywhere. See [9]

Hardy-Littlewood-Wiener theorem states that:

If $f \in L^{1}\left(R^{d}\right)$, there exist $c=c(d)$ such that

$$
\left|\left\{y \in R^{d}: M f(y)>\lambda\right\}\right| \leq \frac{c}{\lambda}\|f\|_{L^{1}\left(R^{d}\right)} \text { for every } \lambda>0
$$

This result indicates that $M f$ maps from $L^{1}\left(R^{d}\right)$ to weak $L^{1}\left(R^{d}\right)$ and $f \in L^{1}\left(R^{d}\right)$ does not claim about $M f \in L^{1}\left(R^{d}\right)$ and thus Hardy-Littlewood maximal operator is not bounded in $L^{1}\left(R^{d}\right)$. In this case we can get only weak type estimates. Hajłasz and Onninen raised same type of question in [15]. Later on Tanaka [6] gave its answer positively for $d=1$.

If $f \in L^{p}\left(R^{d}\right), 1<p \leq \infty$, so is $M f \in L^{p}\left(R^{d}\right)$ then there exist $c=c(d, p)$ such that

$$
\|M f\|_{L^{p}\left(R^{d}\right)} \leq c\|f\|_{L^{p}\left(R^{d}\right)}
$$

This result shows that Hardy-Littlewood operator is bounded in $L^{p}\left(R^{d}\right)$ for $p>1$

Lemma 2: If $v \in C_{0}^{1}\left(R^{d}\right)$, then for every $y \in$ $R^{d}$ and $\omega_{d-1}$, the $(d-1)$-dimensional measure of $\partial B(1,0)$ we have $v(y)=\frac{1}{\omega_{d-1}} \int_{R^{d}} \frac{D v(x) \cdot(y-x)}{|y-x|^{d}} d x$

Proof: We will use fundamental theorem of calculus and start the case by taking one dimensional case.

If $v \in C_{0}^{1}(R)$, then there exists interval $[l, m] \subset R$ and $v(y)=0$ for all $y \in R \backslash[l, m]$

We can write with the help of fundamental theorem of calculus

$$
v(y)=v(l)+\int_{l}^{y} v^{\prime}(x) d x=\int_{-\infty}^{y} v^{\prime}(x) d x
$$

In another way we can write for initial condition $v(l)=0$ as

$$
0=v(m)=v(y)+\int_{y}^{m} v^{\prime}(x) d x=v(y)+\int_{y}^{\infty} v^{\prime}(x) d x,
$$

Or

$$
v(y)=-\int_{y}^{-\infty} v^{\prime}(x) d x
$$

From (3) and (4) we have, $2 v(y)=\int_{-\infty}^{y} v^{\prime}(x) d x-$ $\int_{y}^{\infty} v^{\prime}(x) d x$

$$
=\int_{-\infty}^{y} \frac{v^{\prime}(x)(y-x)}{|y-x|} d x+\int_{y}^{\infty} \frac{v^{\prime}(x)(y-x)}{|y-x|} d x
$$

$$
=\int_{-\infty}^{\infty} \frac{v^{\prime}(x)(y-x)}{|y-x|} d x
$$

Thus $v(y)=\frac{1}{2} \int_{R} \frac{v^{\prime}(x)(y-x)}{|y-x|} d x$ for all $y \in R$

Now, we will extend the result for $R^{n}$,

If $x \in R^{n}$ and $\xi \in \partial B(1,0)$, fundamental theorem of calculus help us to write

$$
v(y)=-\int_{0}^{\infty} \frac{\partial}{\partial t}(v(y+t \xi)) d t=\int_{0}^{\infty} D v(y+t \xi) \cdot \xi d t
$$

Fubini theorem implies,

$$
\begin{aligned}
& \omega_{d-1} v(y)=v(y) \int_{\partial B(1,0)} 1 d S(\xi) \\
= & \int_{\partial B(1,0)} \int_{0}^{\infty} D v(y+t \xi) \cdot \xi d t d S(\xi)
\end{aligned}
$$

Applying Fubini $=-\int_{0}^{\infty} \int_{\partial B(1,0)} D v(y+t \xi) \cdot \xi d S(\xi) d t$

Considering $\quad x=t \xi, d S(\xi)=t^{1-d} d S(x) \quad$ we have $=-\int_{0}^{\infty} \int_{\partial B(1,0)} D v(y+x) \cdot \frac{x}{t} \frac{1}{t^{d-1}} d S(x) d t$

$$
=-\int_{0}^{\infty} \int_{\partial B(1,0)} D v(y+x) \cdot \frac{x}{|x|^{d}} d S(x) d t
$$

In terms of polar coordinates, we can express as,

$$
=\int_{R^{d}} \frac{D v(y+x) \cdot x}{|x|^{d}} d x
$$

replacing $z=y+x, d x=d z=\int_{R^{d}} \frac{D v(z) \cdot z-y}{|z-y|^{d}} d z$

or $=\int_{R^{d}} \frac{D v(x) \cdot y-x}{|y-x|^{d}} d x$

$$
\text { Thus } v(y)=\frac{1}{\omega_{d-1}} \int_{R^{d}} \frac{D v(x) \cdot(y-x)}{|y-x|^{d}} d x
$$

This is the representation formula for a compactly supported continuously differential function in term of its gradient.

By Cauchy-Schwarz inequality and Lemma 2, we can write

$$
\begin{gathered}
|v(y)|=\left|\frac{1}{\omega_{d-1}} \int_{R^{d}} \frac{D v(x) \cdot y-x}{|y-x|^{d}} d x\right| \\
\leq \frac{1}{\omega_{d-1}} \int_{R^{d}} \frac{|D v(x)||y-x|}{|y-x|^{d}} d x \\
\quad \leq \frac{1}{\omega_{d-1}} \int_{R^{d}} \frac{|D v(x)|}{|y-x|^{d-1}} d x \\
\leq \frac{1}{\omega_{d-1}} I_{1}(|D v|)(y)
\end{gathered}
$$

Where $I_{1} f$ denotes the Reisz Potential for $\alpha=1$.

For $0<\alpha<d$, Reisz Potential of order $\alpha$ can be deduced as

$$
I_{\alpha} f(y)=\int_{R^{d}} \frac{f(x)}{|y-x|^{d-\alpha}} d x
$$


One who interested in fundamental properties of Riesz potentials, see e.g. [12].

In case of compactly supported smooth functions, the above result is useful for pointwise bound of functions in term of Reisz potential of the gradient. Some authors have obtained some results for Reisz protentional for example Armin Schikorra and Daniel Spectory [2] established new $L^{1}$-type estimate for Riesz potential. In [3], Petteri Harjulehto, Ritva Hurri-Syrjänen, obtained Pointwise estimates to the modified Riesz potential. Using the similar results, they obtained Poincare Inequality for irregular domain. The inequality of Gagliardo-Nirenberg-Sobolev type was established for nonisotropic Generalized Riesz Potential depending on $\lambda$-distance by Inan Cinar in [18]. Our point of interest here is to discuss boundedness of Reisz Potential by Maximal Operator and then to obtain Gagliardo-Nirenberg inequality

Before moving to the main results, we shall elaborate few technical lemmas for Reisz potential for $\alpha=1$

Lemma 3: If $\psi \subset R^{d}$ is a measureable set and $|\psi|<\infty$, then

$$
\int_{\psi} \frac{1}{|y-x|^{d-1}} d x \leq c(d)|\psi|^{\frac{1}{d}}
$$

Proof: Consider a ball $B=B(y, a)$ with $|B|=|\psi|$. This implies that $|\psi \backslash B|=|B \backslash \psi|$.

We are able to write:

$$
\int_{\psi \backslash B} \frac{1}{|y-x|^{d-1}} d x \leq|\psi \backslash B| \frac{1}{a^{d-1}}
$$

And 2- $|\psi \backslash B| \frac{1}{a^{d-1}} \leq \int_{\psi \backslash B} \frac{1}{|y-x|^{d-1}} d x$ as $|B|=|\psi|$ and $|\psi \backslash B|=|B \backslash \psi|$, then by comparing above inequalities

$$
\begin{gathered}
\int_{\psi} \frac{1}{|y-x|^{d-1}} d x=\int_{\psi \backslash B} \frac{1}{|y-x|^{d-1}} d x \\
\quad+\int_{\psi \cap B} \frac{1}{|y-x|^{d-1}} d x \\
\leq \int_{B \backslash \psi} \frac{1}{|y-x|^{d-1}} d x+\int_{\psi \cap B} \frac{1}{|y-x|^{d-1}} d x \\
=\int_{B} \frac{1}{|y-x|^{d-1}} d x \\
=c(d) a=c(d)|B|^{\frac{1}{d}}=c(d)|\psi|^{\frac{1}{d}}
\end{gathered}
$$

Lemma 4: Let $1 \leq p<\infty$ and assuming $|\Omega|<\infty$ then

$$
\| I_{1}\left(|f| \chi_{\Omega}\left\|_{L^{\left.p_{(}\right)}} \leq c(d, p)|\Omega|^{\frac{1}{d}}\right\| f \|_{L^{p_{(\Omega)}}}\right.
$$

Proof: For $p>1$, by applying Holder inequality, Lemma 3 can be expressed as

$$
\begin{gathered}
\int_{\Omega} \frac{|f(x)|}{|y-x|^{d-1}} d x \\
=\int_{\Omega} \frac{|f(x)|}{|y-x|^{\frac{1}{p}(d-1)}} \frac{1}{|y-x|^{\frac{1}{p^{\prime}(d-1)}}} d x
\end{gathered}
$$

$$
\begin{aligned}
& \leq\left(\int_{\Omega} \frac{|f(x)|^{p}}{|y-x|^{(d-1)}}\right)^{\frac{1}{p}}\left(\int_{\Omega} \frac{1}{|y-x|^{(d-1)}} d x\right)^{\frac{1}{p^{\prime}}} \\
& \leq c|\Omega|^{\frac{1}{d p^{\prime}}}\left(\int_{\Omega} \frac{|f(x)|^{p}}{|y-x|^{(d-1)}} d x\right)^{\frac{1}{p}} \\
& \leq c|\Omega|^{\frac{p-1}{d p}}\left(\int_{\Omega} \frac{|f(x)|^{p}}{|y-x|^{(d-1)}} d x\right)^{\frac{1}{p}}
\end{aligned}
$$

For $p=1$, the above inequality is satisfied. Hence, Using Lemma (3) and Fubini's theorem

$$
\begin{aligned}
\int_{\Omega}\left|I_{1}\left(|f| \chi_{\Omega}\right)(y)\right|^{p} d y & \leq c|\Omega|^{\frac{p-1}{d}} \int_{\Omega} \int_{\Omega} \frac{|f(x)|^{p}}{|y-x|^{(d-1)}} d x d y \\
& \leq c|\Omega|^{\frac{p-1}{d}}|\Omega|^{\frac{1}{d}} \int_{\Omega}|f(x)|^{p} d x
\end{aligned}
$$

This result gives the idea, if $|\Omega|<\infty$, then $I_{1}: L^{p}(\Omega) \rightarrow$ $L^{p}(\Omega)$ is bounded for $1 \leq p<\infty$.

We are now able to discuss the boundedness of Reisz Potential by the Hardy-Littlewood maximal function for general $\alpha$.

Lemma 5: If $0<\alpha<d$, then for every $y \in R^{d}$ and $a>0$, there exist $c=c(d, \alpha)$, such that

$$
\int_{B(y, a)} \frac{|f(x)|^{p}}{|y-x|^{(d-\alpha)}} d x \leq c a^{\alpha} M f(y)
$$

Proof: Let we denote $A_{j}=B\left(y, a 2^{i}\right), i=0,1,2,3, \ldots$

We can express $\int_{B(y, a)} \frac{|f(x)|}{|y-x|^{(d-\alpha)}} d x$

$$
\begin{aligned}
& =\sum_{i=0}^{\infty} \int_{A_{i} \backslash A_{i+1}} \frac{|f(x)|}{|y-x|^{(d-\alpha)}} d x \\
& \leq \sum_{i=0}^{\infty}\left(\frac{a}{2^{i+1}}\right)^{\alpha-d} \int_{A_{i}}|f(x)| d x \\
& =\Omega_{d} \sum_{i=0}^{\infty}\left(\frac{1}{2}\right)^{\alpha-d}\left(\frac{a}{2^{i}}\right)^{\alpha} \frac{1}{\Omega_{d}}\left(\frac{a}{2^{i}}\right)^{-d} \int_{A_{i}}|f(x)| d x \\
& =\Omega_{d} \sum_{i=0}^{\infty}\left(\frac{1}{2}\right)^{\alpha-d}\left(\frac{a}{2^{i}}\right)^{\alpha} \frac{1}{\left|A_{i}\right|} \int_{A_{i}}|f(x)| d x \\
& \leq \operatorname{cMf}(y) a^{\alpha} \sum_{i=0}^{\infty}\left(\frac{1}{2^{\alpha}}\right)^{i}
\end{aligned}
$$

Thus,

$$
\int_{B(y, a)} \frac{|f(x)|}{|y-x|^{(d-\alpha)}} d x \leq c a^{\alpha} M f(y)
$$

Or $I_{\alpha} f(y) \leq c a^{\alpha} M f(y)$

This implies clearly our objective about lemma 5.

With the strong basis of above work and some important results we are going to extend our work toward the main result for sobolev inequality for Reisz potential.

Theorem 1: For $p>1$ and $\alpha>0$ there exists $c=$ 
$c(d, p, \alpha)$, such that for all $f \in L^{p}\left(R^{d}\right)$

We have $\left\|I_{\alpha} f\right\|_{L^{p^{*}\left(R^{d}\right)}} \leq c\|f\|_{L^{p}\left(R^{d}\right)}$,

Where $\alpha p<d$, and $p^{*}=\frac{p d}{d-\alpha p} \mathrm{~F}$
Proof: Observe that the claim is exactly right for $f=0$

Consider $f \neq 0$ on a set of positive measure, it is obvious that $M f>0$ everywhere then

$$
\int_{R^{d} \backslash B(y, a)} \frac{|f(x)|}{|y-x|^{(d-\alpha)}} d x \leq\left(\int_{R^{d} \backslash B(y, a)}|f(x)|^{p} d x\right)^{\frac{1}{p}}\left(\int_{R^{d} \backslash B(y, a)}|y-x|^{(\alpha-d) p^{\prime}} d x\right)^{\frac{1}{p^{\prime}}}
$$

We can compute one part of product on right side as

$$
\begin{aligned}
\int_{R^{d} \backslash B(y, a)}|y-x|^{(\alpha-d) p^{\prime}} d x & =\int_{a}^{\infty} \int_{\partial B(y, \varphi)}|y-x|^{(\alpha-d) p^{\prime}} d S(x) d \varphi \\
& =\int_{a}^{\infty} \varphi^{(\alpha-d) p} \int_{\partial B(y, \varphi)} 1 d S(x) d \varphi
\end{aligned}
$$

Since $\int_{\partial B(y, \varphi)} 1 d S(x) d \varphi=\omega_{d-1} \varphi^{d-1}$, above inequality can be reduced as

$$
\begin{aligned}
\int_{R^{d} \backslash B(y, a)}|y-x|^{(\alpha-d) p^{\prime}} d x & =\omega_{d-1} \int_{a}^{\infty} \varphi^{(\alpha-d) p^{\prime}+n-1} d \varphi \\
\text { Taking integral } & =\frac{\omega_{d-1}}{(\alpha-d) p^{\prime}-d} a^{d-(d-\alpha) p^{\prime}}
\end{aligned}
$$

exponent in (11) can be expressed in the form

$$
\begin{aligned}
d-(d-\alpha) p^{\prime} & =d-(d-\alpha) \frac{p}{p-1} \\
& =\frac{\alpha p-d}{p-1}
\end{aligned}
$$

(11) implies,

$$
\int_{R^{d} \backslash B(y, a)}|y-x|^{(\alpha-d) p^{\prime}} d x=\frac{\omega_{d-1}}{(\alpha-d) p^{\prime}-d} a^{\frac{\alpha p-d}{p-1}}
$$

Applying (11), (10) can rewritten as for any constant $c$,

$$
\int_{R^{d} \backslash B(y, a)} \frac{|f(x)|}{|y-x|^{(d-\alpha)}} d x \leq c a^{\frac{\alpha p-d}{p-1}}\|f\|_{L^{p}\left(R^{d}\right)}
$$

recalling lemma (5), we have $\left|I_{\alpha} f(y)\right| \leq \int_{R^{d}} \frac{|f(x)|}{|y-x|^{(d-\alpha)}} d x$

$$
\begin{aligned}
& =\int_{B(y, a)} \frac{|f(x)|}{|y-x|^{(d-\alpha)}} d x+\int_{R^{d} \backslash B(y, a)} \frac{|f(x)|}{|y-x|^{(d-\alpha)}} d x \\
& \leq c\left(a^{\alpha} M f(y)+a^{\frac{\alpha p-d}{p-1}}\|f\|_{L^{p}\left(R^{d}\right)}\right)
\end{aligned}
$$

Setting $a=\frac{M f(y)}{\left.\|f\|_{L} p_{(R} d\right)} \frac{p}{d}$, we get

$$
\left|I_{\alpha} f(y)\right| \leq c M f(y)^{1-\frac{\alpha p}{d}}\|f\|^{\frac{\alpha p}{d}}{ }_{L^{p}\left(R^{d}\right)}
$$

Taking exponent $p^{*}=\frac{d p}{d-\alpha p}$ on both sides, we have

$$
\left|I_{\alpha} f(y)\right|^{p^{*}} \leq c M f(y)^{p}\|f\|_{L^{p}\left(R^{d}\right)}^{\frac{\alpha p}{d} p^{*}}
$$

By lemma (1),

$$
\int_{R^{d}}\left|I_{\alpha} f(y)\right|^{p^{*}} d x \leq c\|f\|_{L^{p}\left(R^{d}\right)}^{\frac{\alpha p}{d} p^{*}} \int_{R^{d}} M f(y)^{p} d y
$$

$$
\begin{aligned}
& \leq c\|f\|_{L^{p}\left(R^{d}\right)}^{\frac{\alpha p}{d} p^{*}}\|M f\|_{L^{p}\left(R^{d}\right)}^{p} \\
& \leq c\|f\|_{L^{p}\left(R^{d}\right)}^{\frac{\alpha p}{d} p^{*}}\|f\|_{L^{p}\left(R^{d}\right)}^{p}
\end{aligned}
$$

Hence, $\left\|I_{\alpha} f\right\|_{L^{p^{*}\left(R^{d}\right)}} \leq c\|f\|_{L^{p}\left(R^{d}\right)}^{\frac{\alpha p}{d}+\frac{P}{p^{*}}}$

$$
\leq c\|f\|_{L^{p}\left(R^{d}\right)}
$$

Here $p^{*}=\frac{p d}{d-\alpha p}$ is the Sobolev conjugate if $\alpha=1$. Also, for $p=1$ we can produce weak type estimates.

From (14), for $p=1$ there exist $c=c(d, \alpha)$ such that

$$
\left|I_{\alpha} f(y)\right| \leq c M f(y)^{1-\frac{\alpha}{d}}\|f\|^{\frac{\alpha}{d}}{ }_{L^{1}\left(R^{d}\right)}
$$

taking maximal function approach for $p=1$, we have

$\left|\left\{y \in R^{d}:\left|I_{\alpha} f(y)\right|>t\right\}\right|$

$$
\begin{aligned}
& \leq\left|\left\{y \in R^{d}: M f(y)>c t^{\frac{d}{d-\alpha}}\|f\|_{L^{1}\left(R^{d}\right)}^{-\frac{\alpha}{d} \cdot \frac{d}{d-\alpha}}\right\}\right| \\
& \leq c t^{-\frac{d}{d-\alpha}\|f\|_{L^{1}\left(R^{d}\right)}^{\frac{\alpha}{d-\alpha}}\|f\|_{L^{1}\left(R^{d}\right)}}
\end{aligned}
$$

Hence $\left|\left\{y \in R^{d}:\left|I_{\alpha} f(y)\right|>t\right\}\right| \leq c t^{-\frac{d}{d-\alpha}}\|f\|_{L^{1}\left(R^{d}\right)}^{\frac{\alpha}{d-\alpha}}$

This can also give for every $t>0$,

$$
\left|\left\{y \in R^{d}:\left|I_{\alpha} f(y)\right| \geq t\right\}\right| \leq c t^{-\frac{d}{d-\alpha}}\|f\|_{L^{1}\left(R^{d}\right)}^{\frac{\alpha}{d-\alpha}}
$$

It will help us to deduce the proof Sobolev GagliardoNirenberg Inequality.

\section{Main Result}

Theorem 2: For every, $v \in C_{0}^{1}\left(R^{d}\right)$, if $1 \leq p<d$, there exist $c=c(d, p)$ such that

$$
\|v\|_{L^{p^{*}\left(R^{d}\right)}} \leq c\|D v\|_{L^{p}\left(R^{d}\right),} p^{*}=\frac{p d}{d-p}
$$

Proof: 
We will split the proof into two cases.

Case I: First we will take the case for $p>1$

For every, $y \in R^{d}$, we can write from (1.7),

$\|v(y)\| \leq \frac{1}{\omega_{d-1}} I_{1}(|D v|)(y)$ where $1<p<d$

Changing the inequality for $L^{p^{*}}\left(R^{d}\right)$,

$$
\|v\|_{L^{p^{*}}\left(R^{d}\right)} \leq c\left\|I_{1}(|D v|)\right\|_{L^{p^{*}}\left(R^{d}\right)}
$$

By using above theorem (6) for $\alpha=1$, we can write

$$
\|v\|_{L^{p^{*}}\left(R^{d}\right)} \leq c\|D v\|_{L^{p}\left(R^{d}\right)}
$$

This is the required inequality for $p>1$

Case II: For $p=1$, let we consider pairwise disjoint sets like

$B_{i}=\left\{y \in R^{d}: 2^{i}<|v(y)| \leq 2^{i+1}\right\}, i \in Z$

Suppose $g: R \rightarrow R, g(t)=\max \{0, \min \{t, 1\}\}$, be an auxiliary function and $v_{i}: R^{d} \rightarrow[0,1]$,

$$
\begin{aligned}
v_{i}(x) & =g\left(2^{1-i}|v(y)|-1\right) \\
& =\left\{\begin{array}{c}
0, \quad|v(y)| \leq 2^{i-1} \\
2^{1-i}|v(y)|-1, \quad 2^{i-1}<|v(y)| \leq 2^{i} \\
1, \quad|v(y)|>2^{i}
\end{array}\right.
\end{aligned}
$$

Using lemma, if $v_{i} \in W^{1,1}\left(R^{d}\right)$, and $i \in Z$ then $D v_{i}=0$ almost a.e. in $R^{d} \backslash B_{i-1}$.

We can write, $\left|B_{i}\right| \leq\left|\left\{y \in R^{d}:|v(y)| \leq 2^{i}\right\}\right|$

if $|v(y)| \leq 2^{i}$ it implies that $2^{1-i}|v(y)|-1>1$, above result can be written as

$$
\begin{aligned}
\left|B_{i}\right| & =\left|\left\{y \in R^{d}: v_{i}(y)=1\right\}\right| \\
& \leq\left|\left\{y \in R^{d}: I_{1}\left(\left|D v_{i}\right|\right)(y) \geq \omega_{d-1}\right\}\right|, \text { by applying } \\
& \leq c\left(\int_{R^{d}}\left|D v_{i}(y)\right| d y\right)^{\frac{d}{d-1}}, \text { by applying (15) } \\
& \leq c\left(\int_{B_{i-1}}\left|D v_{i}(y)\right| d y\right)^{\frac{d}{d-1}} \\
& \leq c\left(\int_{B_{i-1}} g^{\prime}\left(2^{1-i}|v(y)|-1\right) 2^{1-i}|D v(y)| d y\right)^{\frac{d}{d-1}} \\
& \leq c 2^{-i \frac{d}{d-1}}\left(\int_{B_{i-1}}|D v(y)| d y\right)^{\frac{d}{d-1}}
\end{aligned}
$$

Applying summation for $i \in Z$, we get

$$
\begin{aligned}
\int_{R^{d}}|v(y)|^{\frac{d}{d-1}} & =\sum_{i \in Z} \int_{B_{i}}|v(y)|^{\frac{d}{d-1}} d y \\
& \leq \sum_{i \in Z} 2^{(i+1) \frac{d}{d-1}}\left|B_{i}\right| \\
& \leq c \sum_{i \in Z}\left(\int_{B_{i-1}}|D v(y)| d y\right)^{\frac{d}{d-1}} \\
& \leq c\left(\sum_{i \in Z} \int_{B_{i-1}}|D v(y)| d y\right)^{\frac{d}{d-1}}
\end{aligned}
$$

Hence $\int_{R^{d}}|v(y)|^{\frac{d}{d-1}} \leq c\left(\int_{R^{d}}|D v(y)| d y\right)^{\frac{d}{d-1}}$

\section{Remark}

By considering the fact that $C_{1}^{0}\left(R^{d}\right)$ is dense in $W^{1, p}\left(R^{d}\right)$ then for $v \in W^{1, p}\left(R^{d}\right)$, the Sobolov-Gagliardo-Nirenberg inequality follows from the above theorem where $1 \leq p<n$.

\section{References}

[1] N. Kalton, S. Mayboroda, and M. Mitrea, Interpolation of Hardy-Sobolev-Besov-Triebel-Lizorkin spaces and applications to problems in partial differential equations, pp. 121-177 in Interpolation theory and applications (Miami, FL, 2006), edited by L. De Carli and M. Milman, Contemp. Math. 445, Amer. Math. Soc., Providence, RI, 2007.

[2] Armin Schikorra, Daniel Spectory: An $L^{1}$-type estimate for Riesz potentials, Revista Matematica Iberoamericana 33 (2017), no. 1, 291-304.

[3] Petteri Harjulehto, Ritva Hurri-Syrjänen, Pointwise estimates to the modified Riesz potential, manuscripta math. 156, 521543 (2018).

[4] Hajłasz, P., and Z. Liu: Sobolev spaces, Lebesgue points and maximal functions.-J. Fixed Point Theory Appl. 13: 1, 2013, 259-269.

[5] Gilbarg D., and N. S. Trudinger: Elliptic Partial Differential Equations of Second Order.-Springer-Verlag, Berlin, 1983.

[6] Tanaka, H.: A remark on the derivative of the one-dimensional Hardy-Littlewood maximal function.-Bull. Austral. Math. Soc. 65: 2, 2002, 253-258.

[7] P. Haj lasz, A new characterization of the Sobolev space, Studia Math. 159 (2003), no. 2, 263-275.

[8] Maz'ya, V.: Sobolev spaces with applications to elliptic partial differential equations. Second, revised and augmented edition. Grundlehren der Mathematischen Wissenschaften [Fundamental Principles of Mathematical Sciences], 342. Springer, Heidelberg, 2011.

[9] P. Koskela and E. Saksman, Pointwise characterizations of Hardy-Sobolev functions, Math. Res. Lett. 15 (2008), no. 4, 727-744.

[10] J. M. Aldaz and J. P erez L azaro, Functions of bounded variation, the derivative of the one-dimensional maximal function, and applications to inequalities, Trans. Amer. Math. Soc. 359 , no. 5 (2007), 2443-2461.

[11] N. Kalton, S. Mayboroda, and M. Mitrea, Interpolation of Hardy-Sobolev-Besov-Triebel-Lizorkin spaces and applications to problems in partial differential equations, pp. 121-177 in Interpolation theory and applications (Miami, FL, 2006), edited by L. De Carli and M. Milman, Contemp. Math. 445, Amer. Math. Soc., Providence, RI, 2007.

[12] Y. Mizuta, Potential theory in Euclidean spaces, Gakk_otosho, Tokyo, 1996.

[13] Luiro, H.: Continuity of maximal operator in Sobolev spaces. Proc. Am. Math. Soc. 135, 243-251 (2007). 
[14] Carlos p erez, Tiago picon, Olli saari, regularity of maximal functions on hardy-sobolev spaces, Mathematics Subject Classification. 2010, 42B25, 42B30, 46E35.

[15] P. Hajlasz and J. Onninen, on boundedness of maximal functions in sobolev spaces, Annales Academie Scientiarum Fennicae Mathematica, Volume 29, 2004, 167-176.

[16] Ondřej Kurka, on the variation of the hardy-Littlewood maximal function, Annales Academiæ Scientiarum Fennicæ
Mathematica, Volumen 40, 2015, 109-133.

[17] G. Leoni, A First Course in Sobolev Spaces, AMS, 2009.

[18] Inan Cinar, The Gagliardo-Nirenberg-Sobolev Inequality for Non-Isotropic Riesz Potentials, Journal of Mathematics and System Science 5 (2015) 83-85. 